\title{
Multimodale Praktiken der Konstruktion von Emotionen in printmedialen Diskursen ${ }^{1}$
}

\author{
Multimodal practices of the construction \\ of emotions in print media discourses \\ Jacek SZCZEPANIAK ${ }^{2}$ \\ Uniwersytet Kazimierza Wielkiego w Bydgoszczy
}

\begin{abstract}
Zusammenfassung
Ziel des vorliegenden Beitrags ist es zu zeigen, wie Emotionen als interindividuelle, intersemiotische Entitäten, als relevante Diskursfaktoren, als „Bestandteile von routinisierten, kulturell standardisierten Praktikenkomplexen“ (Reckwitz, 2016, S. 173) konzipiert werden können. Diskurse, darunter auch massenmediale, werden hier als transtextuelle und transmodale Gebilde angesehen, die Orte der Entstehung, Manifestation bzw. Generierung von Emotionen sind. Gegenstand der Analyse sind Text-Bild-Konglomerate printmedialer Kommunikation (Titelseiten von polnischen Nachrichtenmagazinen), die in Hinblick auf ihre Emotions- bzw. Emotionalisierungspotenziale untersucht werden.

Schlüsselwörter: Emotion, Multimodalität, Medialität, Diskurs, linguistische Diskursanalyse
\end{abstract}

\begin{abstract}
The aim of this paper is to present how emotions can be conceptualized as interindividual, intersemiotic entities, as relevant discourse factors, as "components of routinized, culturally standardized practice complexes" (Reckwitz, 2016, p. 173). Discourses, including mass media ones, are seen here as transtextual and transmodal entities that are sites of emergence.
\end{abstract}

\footnotetext{
1 Dieser Artikel entstand im Rahmen des Projekts „Emotionale Nachbarschaft. Affekte in deutsch-polnischen medialen Diskursen seit dem EU-Beitritt Polens“, welches mit Mitteln der Deutsch-Polnischen Wissenschaftsstiftung gefördert wird.

2 (D)0000-0001-7009-7168

Uniwersytet Kazimierza Wielkiego w Bydgoszczy

jacek.szczepaniak@ukw.edu.pl
} 
Keywords: emotion, multimodality, mediality, discourse, linguistic discourse analysis

\section{Einführende Bemerkungen}

Ohne schriftliche Ausdrucksmittel und ikonische Elemente als „externalisierte Instrumente gesellschaftlicher Interaktion [...] gibt es keine identifizierbaren Gedanken“, schreibt Dietrich Busse (2016, S. 648). Diese Diagnose kann ebenso auf Emotionen als Kommunikationsphänomene bezogen werden, die - wie viele andere anthropologische, soziale, politische, historische usw. Sachverhalte - medial fundiert sind. Dass jede Artikulation notwendig ein Medium voraussetze, ist in Kommunikations- und Medienwissenschaften mittlerweile ein Gemeinplatz. Demnach gibt es in der Kommunikation auch kein außermediales Funktionieren von Emotionen. Mit der Medialität der Emotion sind materiale, medial bedingte (sprachliche und extrasprachliche) Rahmenbedingungen: Formen, Strukturen und Mittel der Manifestation und Generierung von Emotionen gemeint. Mittels Zeichensysteme unterschiedlicher semiotischer Provenienz werden Emotionen evoziert, hergestellt, vermittelt, gespeichert, modifiziert und multipliziert. Erst durch die je spezifische Materialität der jeweiligen Zeichen bekommen sie ihre Identität, Abrufbarkeit und Wiederholbarkeit in der Kommunikation sowie können diskursiv hervorgebracht und medial vermittelt werden. Die semiotisch orientierten Dimensionen der Kommunikation lenken demnach den Blick einer medien- und diskurslinguistisch fundierten Emotionsforschung auf die Multimodalität und intersemiotischen Relationen, die zur Subtilität und Vieldeutigkeit, aber auch zur Ausdrucksstärke und Intensität emotionaler Prozesse beitragen (vgl. Schiewer, 2014, S. 42). Deswegen sollen die „komplexen, sich wechselseitig bedingenden, miteinander interagierenden Verhältnisse zwischen Sichtbarem und Sagbarem ins analytische Visier" genommen werden (Massen, Mazerahuser \& Renggli, 2006, S. 8).

Will man Emotionen aus kultur-, medien- oder sprachwissenschaftlicher Sicht analysieren, reicht es somit nicht aus, nur verbale Elemente und Möglichkeiten ihrer Verwendung in kommunikativen Praktiken in den Blick zu nehmen. Komplexe Zeichenkonglomerate, wie z.B. Emotionen, entstehen nämlich durch die Verknüpfung verschiedener Modi. In printmedialem Umfeld beispielsweise sind das visuelle Modi: Schriftsprache 
und Bild(er $)^{3}$, die als zentrale Ressourcen der Konstruktion von Wissen (und auch Emotionen) in massenmedialen Diskursen aufzufassen sind. Deswegen integriert auch die Diskurslinguistik die Untersuchung von semiotisch komplexen Artefakten und somit von Text-Bild-Relationen in ihre Analysemodelle (vgl. z. B. Felder, 2012, S. 142; Bendel Larcher, 2015, S. 127ff. $)^{4}$. Im 2017 von Ernest W. B. Hess-Lüttich, Heidrun Kämper, Martin Reisigl und Ingo H. Warnke veröffentlichten Sammelband „Diskurs semiotisch" wird explizit auf die Komplexität kommunikativer Praktiken und deren Multimodalität verwiesen. Es wird betont, dass diese Komplexe von Zeichensystemen „aufeinander verweisen und miteinander Sinn konstituieren, in ihrer diskursiven Geprägtheit“. Zu exponieren sind in diesem Zusammenhang „diskursive Codierungen und ihre mutuellen Beziehungen als Bedeutung schaffende Instanzen“ (Hess-Lüttich et al., 2017, S. IX). Der Diskurs kann somit als ein semiotisches Kohärenzphänomen wie auch „als sozialer und historischer Ort von Wissen" (Spitzmüller \& Warnke, 2011, S. 67) konzipiert werden.

Der vorliegende Beitrag verfolgt das Ziel, aus einer sprachgebrauchsorientierten und mediensensiblen Perspektive zu zeigen, wie Emotionen als interindividuelle, intersemiotische Entitäten, als relevante Diskursfaktoren, als „Bestandteile von routinisierten, kulturell standardisierten Praktikenkomplexen“ (Reckwitz, 2016, S. 173) konzipiert werden können. Diskurse, darunter auch massenmediale, werden hier als transtextuelle und transmodale Gebilde angesehen, „die sich über das singuläre Zeichensystem der Sprache hinaus konstituieren“ (Klug, 2018, S. 109) und die Orte der Entstehung, Manifestation bzw. Generierung von Emotionen sind. Das Erkenntnisinteresse richtet sich dabei auf bestimmte schriftsprachliche und ikonische Konventionen bzw. Ordnungen der Manifestation und Generierung von Emotionen im printmedialen Umfeld, die in einem konkreten Praxiszusammenhang entstehen.

Der vorgeschlagene Ansatz ermöglicht es, Emotionen in ihrer jeweiligen medialen, vorwiegend multimodalen Gestalt als situierte, historisch und soziokulturell geprägte Konstrukte, als Resultate sozialer und medialer

\footnotetext{
${ }^{3}$ Aus semiotischer Sicht betrachtet Sachs-Hombach (2001, S. 11) Bilder als „wahrnehmungsnahe Zeichen".

4 In diesem Zusammenhang konstatieren Eckkrammer und Held (2006, S. 2), dass „nur die Semiotik jenen Leitrahmen vorgeben kann, der in der Lage ist, die Gleichrangigkeit der Zeichen - als semiotische Ressourcen oder Modes - zumindest heuristisch zu gewährleisten“.
} 
Interaktionen $\mathrm{zu}$ analysieren, und macht zugleich ihre kommunikative Relevanz sichtbar.

Gegenstand der Analyse sind Text-Bild-Konglomerate printmedialer Kommunikation - ausgewählte Titelseiten von polnischen Nachrichtenmagazinen mit rechtskonservativem Profil -, die in Hinblick auf ihre Emotions- bzw. Emotionalisierungspotenziale untersucht werden. Entscheidend bei der Auswahl war die Diskurszugehörigkeit einzelner TextBild-Konglomerate, die sich u.a. durch die Wiederaufnahme des Themas (deutsch-polnische Geschichte) und der Akteure (deutsche Politiker*innen) erkennen lassen.

\section{Diskursanalyse als Methode der linguistischen Emotionsforschung}

Die Aufgabe einer Diskursanalyse liegt in der Erforschung von Diskursen als Praktiken, die systematisch Gegenstände bilden, von denen sie sprechen (vgl. Foucault, 1981, S. 74). Ihr Ziel ist es, zur „Sichtbarmachung der diskursiv ermittelten Wirklichkeit“ (Teubert, 2013, S. 64) ihren Beitrag zu leisten. Denn die Aussage im Diskurs ist der Ort, an dem die wirklichkeitsund wissenskonstitutive Kraft von Sprache - in Kooperation mit anderen semiotischen Zeichensystemen - wirksam und erkennbar wird.

Diskursanalysen foucaultscher Provenienz verfolgen im Allgemeinen das Ziel, die Beziehungen von Elementen zu untersuchen. Sie beschreiben somit das Zusammenbringen von Elementen als diskursives In-Beziehung-Setzen und zeigen, wie auf diese Weise eine Welt bzw. Wirklichkeit konstruiert wird (vgl. Renngli, 2014, S. 49). Durch die Beschreibung diverser Relationen zwischen Bausteinen eines Diskurses oder Komponenten verschiedener Diskurse sowie nichtdiskursiver Praktiken, d.h. intra-, inter- und extradiskursiver Dependenzen, soll im Sinne Foucaults „das polymorphe Bündel von Zusammenhängen“ hervortreten (Foucault 2001b, S. 867 f.). Eine Diskursanalyse, die auf Foucaults Konzeptionen aufbaut, kann sich somit nicht auf Texte im Sinne einer monokodierten Sprachlichkeit beschränken ${ }^{5}$. Diese existiert eigentlich kaum, da selbst die Schriftsprache multimodale Züge aufweist, z. B. die Typographie der Schrift, ihre Farbe oder das grafische Layout

\footnotetext{
${ }^{5}$ Zum Zusammenhang von Bild und Sprache (Text) hat sich auch Michel Foucault mehrmals geäußert, allerdings mit Bezug auf bildende Kunst. So konstatiert er: „Diskurs und Figur haben jeweils ihre eigene Seinsweise; aber sie unterhalten komplexe, verschachtelte Beziehungen. Ihr wechselseitiges Funktionieren gilt es zu beschreiben." (Foucault, 2001a, S. 796)
} 
(mehr dazu z. B. Spitzmüller, 2013, S. 153-158). Über den Text hinaus ist somit auch die schriftsprachliche Kommunikation „ein visuelles Arrangement der Schriftzeichen auf einer Fläche als Gestaltungseinheit" (Bucher, 2012, S. 55). Es ist also davon auszugehen, dass an der Sinnkonstitution von Texten als Diskursausschnitten verschiedene Kommunikationsmodi multiplikatorisch zusammenwirken.

Der medien- und diskurslinguistische Ansatz in der Emotionsforschung geht der Frage der Emotionalisierung von diskursiven Ereignissen nach und berücksichtigt bei der Untersuchung Aspekte, die den Zusammenhang zwischen Emotionen, semiotischen Zeichen und medialen Diskursen offenbaren. Ausgehend von der Tatsache, dass Emotionen, Medien und Diskurse wechselseitig miteinander verschränkt sind, stellt sich nun die Frage, wie Emotionen als kommunikative Erscheinungen in Diskursen manifestiert bzw. generiert werden. Emotionen in ihrer medialen (materiellen) Gestalt stellen das Ergebnis unterschiedlicher diskursiver Prozesse dar, das sich aus dem Zusammenspiel diverser Faktoren ergibt.

\section{Emotionen als diskursive Konstrukte}

Den vorliegenden Ausführungen liegt die Annahme zugrunde, dass emotionale Kommunikation einen Artefaktcharakter hat: Emotionen als soziale und kulturelle Phänomene weisen immer ein kontextgebundenes und medienspezifisches Design, ihre je eigene/spezifische Medialiät auf. So konzipierte Emotionen stellen „ein von menschlicher Praxis reguliertes Geschehen unseres gesellschaftlichen Lebens" (Gebauer et. al, 2017, S. 9) dar: Sie können von individuellen oder kollektiven Akteuren, die über ein gemeinsames, diskursiv generiertes Emotionswissen verfügen, konstruiert, wahrgenommen, beobachtet, interpretiert oder thematisiert werden. Wie im Falle einer jeden sozialen (diskursiven) Praktik bildet das gemeinsame Wissen zwischen den Diskursakteuren also auch bei Emotionen die Basis für erfolgreiches Kommunizieren. Auf dieser Grundlage kann der Zusammenhang zwischen dem explizit Geäußerten und dem implizit Gemeinten rekonstruiert werden. Emotionen als semiotische Entitäten und soziale Handlungspraktiken zugleich können jenen Tätigkeiten zugerechnet werden, die sich als ein Operieren mit Zeichen auffassen lassen. Es bedarf bestimmter Fertigkeiten, „Techniken“ - Bestandteile der semiotischen Kompetenz -, die es möglich machen, von sinnlich wahrnehmbaren Elementen auf nicht direkt Wahrnehmbares zu schließen. Keller (2003, S. 173) spricht 
in diesem Zusammenhang vom „Interpretieren“ und verweist darauf, dass „die Basis der interpretativen Schlüsse [...] die Gebrauchsregeln der verwendeten Ausdrücke“ bilden. Im gerahmten Kontext einer diskursiven Praxis können somit die Kommunizierenden mit Hilfe unterschiedlicher Zeichensysteme „aus dem nicht-faßbaren Fließen erkennbare Muster bilden“ (Gebauer, 2009, S. 182).

Die Überlegung, die Medialitätsebene als Dimension der Kodierung und/oder Generierung von Emotionen im Diskurs zu betrachten, ergibt sich aus der Tatsache, dass Medien - als "Verfahren der Zeichenprozessierung" (Schneider, 2017, S. 37) begriffen - unhintergehbar zeichenhaft sind. Daher steht im Mittelpunkt des analytischen Interesses die Medialität, „d.h. die medialen Eigenschaften des jeweiligen medialen Verfahrens" (Schneider, 2017, S. 37). Im Rahmen einer bestimmten sozialen Praxis und in Kooperation von Zeichen unterschiedlicher Modalitäten - schriftsprachlicher mit ikonischen Elementen (Bild, Typographie) - werden emotionale Bedeutungen generiert. Text-Bild-Verflechtungen bzw. (intersemiotische) Zusammenspiele von Schrift(sprache) und Bild werden in diesem medialitätsorientierten Ansatz als Konstitutionsbedingungen diskursiver Praxis betrachtet, als geeignete Instrumente zur Manifestation und Generierung von Emotionen.

Die Feststellung, dass schriftsprachliche Texte in der massenmedialen Kommunikation in der Regel nicht isoliert vorkommen, sondern in Kombination mit Artefakten anderer semiotischer Provenienz wie Bildern, Fotos, Diagrammen, auch Musik oder Geräuschen usw., ist mittlerweile ein Gemeinplatz. Massenmediales Kommunizieren vollzieht sich in vielfältiger Materialisierung, was sich im Einsatz verschiedenster Modi niederschlägt. Daher sind auch Diskurse als multimodale Größen, genauer: als multimodal konstruierte und realisierte Artikulationen von Wissensräumen zu betrachten: Bezüglich ihrer Konstitutionsmechanismen umfassen sie „institutionellorganisatorisch regulierte" Praktiken ${ }^{6}$ des sprachlichen und nichtsprachlichen Zeichengebrauchs, in deren Rahmen „von gesellschaftlichen Akteuren im Sprach- bzw. Symbolgebrauch die soziokulturelle Bedeutung und Faktizität physikalischer und sozialer Realitäten konstituiert [wird]“ (Keller, 2013, S. 27).

\footnotetext{
${ }^{6}$ Nach Reiner Keller werden diskursive Praktiken wie folgt konzeptualisiert: „Als diskursive Praktiken bezeichne ich typische realisierte Kommunikationsmuster, sofern sie in einem Diskurszusammenhang eingebunden sind. [...] Diskursive Praktiken sind beobachtbare und beschreibbare typische Handlungsweisen der Kommunikation, deren Ausführung als konkrete Handlung - ähnlich wie im Verhältnis zwischen typisierbarer Aussage und konkret-singulärer Äußerung - der interpretativen Kompetenz sozialer Akteure bedarf und von letzteren aktiv gestaltet wird. (Hervorhebungen im Original; JS)“ (Keller, 2005, S. 223).
} 
An diskursiver Wissenserzeugung und folglich an der (Re)Produktion von Diskursen können Aktivitäten verschiedener Art und alle Phänomene beteiligt sein, denen Bedeutung in Kommunikationsprozessen zugeschrieben wird (vgl. z. B. Knoblauch \& Tuma, 2018, S. 517).

Emotionen als kommunikative Phänomene und multimodale Konstrukte können demnach im Rahmen einer multimodal ausgerichteten Diskursanalyse untersucht werden, da diese Forschungsperspektive "Sensibilität für die kommunikativen Signifikationsvollzüge [schafft], die durch bestimmte Gestaltung der Zeichenausdrucksebene und der Sinnkonstruktion auf der Zeicheninhaltsebene der Diskursfragmente realisiert sind“ (Meier, 2011, S. 504). Die multimodale Zeichenhaftigkeit von Emotionen kann dadurch erfasst und analysiert werden, dass ihr soziokultureller Gebrauch und ihre mediale Kontextualisierung fokussiert werden

\section{Multimodale Praktiken der Konstruktion von Emotionen - Beispiele}

,Multimodalität' wird mit Fricke (2012, S. 37) als medial basiertes Phänomen der funktionalen Integration mehrerer Codes in einem gemeinsamen Primärbzw. Meta-Code aufgefasst. Multimodale Gefüge lassen sich entsprechend als kommunikativ angelegte, rezeptions- und deutungsoffen gesetzte intersemiotische Relationen beschreiben, für die das Merkmal der „Übersummativität“ als basal (vgl. Igl \& Menzel, 2016, S. 14) anzusetzen ist:

Als semiotische Systeme basieren 'Bild und 'Sprache zwar auf einem Repräsentationsverhältnis zwischen Zeichen und Welt; es wäre aber stark verkürzt, sie lediglich als mediale Informationsträger zu sehen. Das bedeutet im Gegenzug zugleich, dass Bildern im Rahmen von Text-Bild-Gefügen nicht einfach eine kumulativ-illustrierende Funktion zugeschrieben werden kann, sondern dass diese gleichermaßen wie der Text bzw. die Sprachzeichen an der Konstitution von Bedeutung beteiligt sind. (Igl \& Menzel, 2016, S. 17)

In der heutigen (massen)medial geprägten Gesellschaft sehen wir uns permanent mit visuellen Artikulationsformen konfrontiert - typographisch unterschiedliche Schrifttexte, Abbildungen, Zeichnungen und Diagramme sind omnipräsente Komponenten der modernen Lebenswelt. Diese Artefakte sind Produkte visueller Praktiken - relevanter Verfahrensweisen zur Generierung und Vermittlung des Wissens in modernen Informations- und zugleich Mediengesellschaften (vgl. z. B. Sachs-Hombach \& Schirra, 2009, S. 393). 
Zur Veranschaulichung der obigen Ausführungen wird hier aus Platzgründen eine geringe Datengrundlage (zwei Covers) herangezogen. Entscheidend bei der Auswahl war - wie bereits erwähnt - die Diskurszugehörigkeit einzelner Text-Bild-Konglomerate.

1) „Gazeta Polska Codziennie“ - Titelseite der Ausgabe vom 05.-06.01. 2016

Den deutschen Überfall auf Polen 1939 symbolisiert kein anderes Foto so sehr wie die vom Danziger Fotografen Hans Sönnke gemachte Aufnahme mit den neun Uniformierten, die sich gegen einen Schlagbaum stemmen.

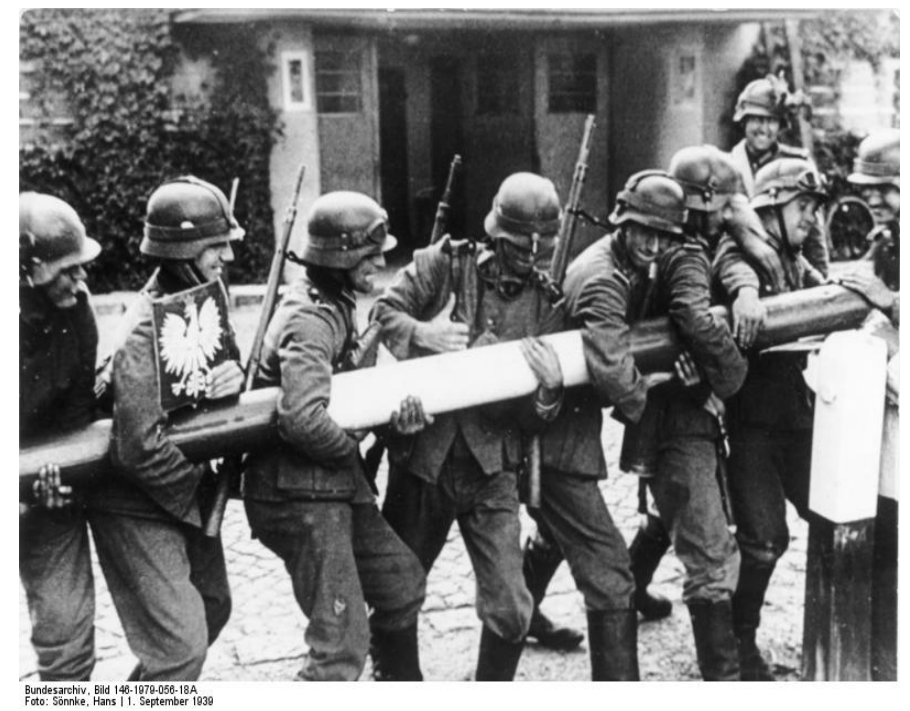

Abb. 1: File:Bundesarchiv Bild 146-1979-056-18A, Polen, Schlagbaum, deutsche Soldaten.jpg - Wikimedia Commons [Zugriff: 20.10.2021]

Die nationalkonservative Tageszeitung "Gazeta Polska Codziennie“ brachte vor ein paar Jahren ein Titelbild, auf dem Gesichter von deutschen und EU-Politiker*innen ${ }^{7}$ in ein historisches Foto vom September 1939 montiert wurden, u. z. unter dem Titel: „Die Deutschen greifen Polen wieder an“.

\footnotetext{
7 Gemeint sind: Günther Oettinger (der damalige EU-Kommisar für Energie), Martin Schulz (der damalige Präsident des Europäischen Parlaments) und Angela Merkel (Bundeskanzlerin).
} 


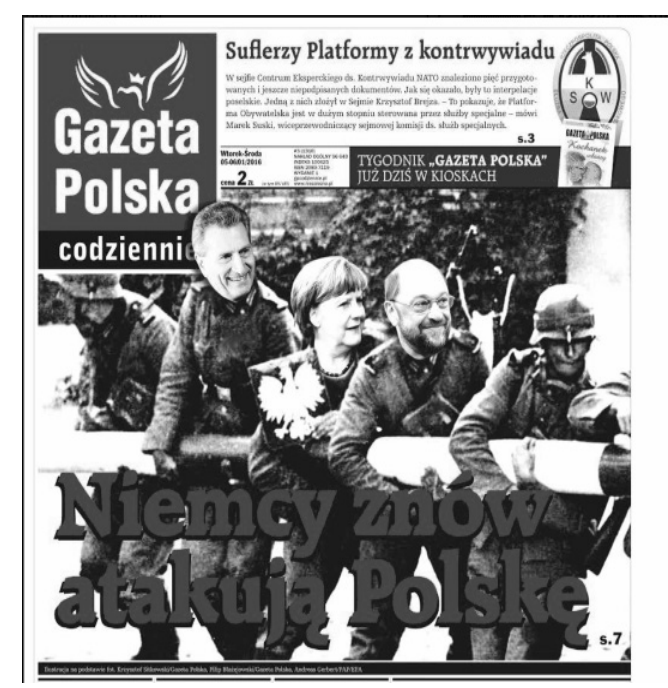

Abb. 2. https://www.facebook.com/GPCodziennie/photos/a.263232313698405/ 1046688722019423 [Zugriff: 20.10.2021]

Das emotionale Potenzial des Bildes wird aktiviert und intensiviert durch die Kooperation mit dem Text, da bestimme Assoziationen hervorgerufen werden. Im vorliegenden Fall werden Emotionen mittels den Strategien der Interikonizität und der Intertextualität sowie intermodalen Relationen als Diskursmuster evoziert. Dadurch steigt auch das „Aktivierungspotenzial“ dieses multimodalen Kommunikats, d.h. die Fähigkeit, Aufmerksamkeit auszulösen. Die Wahrnehmung des Bildes und seine kognitive Verarbeitung (in Kombination mit Sprachtext und diskursivem Wissen des/der Rezipierenden) kann bzw. sollte zur Folge haben, dass bestimmte Assoziationen bzw. Bilder aus dem kollektiven Gedächtnis aktiviert und diskursmotiviert handelnde Bedeutungen wie Emotionen bzw. Affekte gestiftet werden (vgl. Meier, 2011, S. 519). „Das Wissen um die [... aufmerksamkeitsfesselnde Wirkung von Bildern ist mittlerweile Allgemeinwissen der Werbebranche in ihren politischen und kommerziellen Zweigen" - konstatiert Traue (2014, S. 137). Ihren Ausdruck findet sie eben in überraschenden, oft schockierenden, emotionsgeladenen Bildeinsetzen, Kollagen usw.

Text-Bild-Konglomerate sind deutlich effektiver in Generierung von Emotionen, da ihnen nicht die argumentative Logik rein textueller Kommunikation zugrunde liegt, sondern assoziative Logik, die typisch für Bilder ist. Bilder knüpfen so an innere Bilder der Betrachter an und lösen bestimmte Assoziationsketten aus. 
2) „Uważam Rze” - Titelseite der Ausgabe vom 07.04. 2014

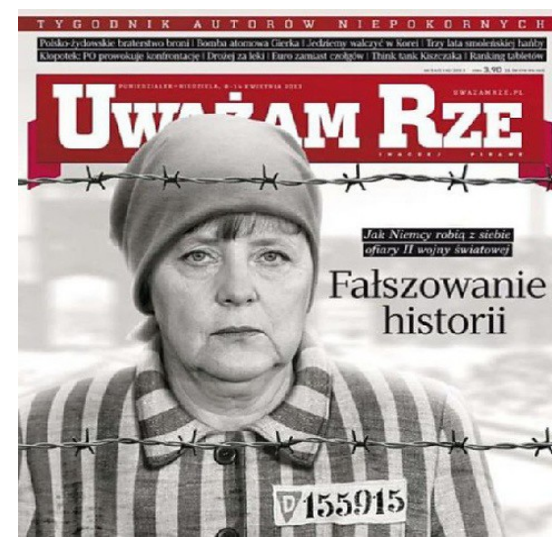

Abb. 3 http://www.old.uwazamrze.pl/miesiecznik/1447 [Zugriff: 20.10.2021]

Das präsentierte Cover bezieht sich thematisch auf die Debatte um den ZDF-Dreiteiler „Unsere Mütter, unsere Väter"8 (2013), der für große Kontroversen und heftige Diskussionen in polnischen Massenmedien gesorgt hat. Die drei Teile der Kurzserie wurden im März 2013 im ZDF und im ORF ausgestrahlt. Die vielgelobte ${ }^{9}$ Kurzserie wurde vor allem in Polen sehr stark kritisiert: Es ging vor allem um die Darstellung der Heimatarmee und polnischer Partisanen, die gegen die NS-Besatzer kämpften und die im Film deutliche antisemitische Tendenzen zeigten.

Nun reagierte darauf das polnische rechtskonservative Magazin „Uważam Rze“ mit einer Fotomontage auf dem Cover. Es zeigte die deutsche Bundeskanzlerin Angela Merkel hinter einem Stacheldrahtzaun in der Kleidung eines KZ-Häftlings. Im Hintergrund zeichnete sich der Umriss eines Konzentrationslagers ab. Das ausdrucksstarke Bild begleitet die Überschrift: „Geschichtsfälschung“.

Derartige kraftvolle Text-Bild-Kompositionen können narrative Strukturen schaffen. In Hinblick auf die beiden gezeigten Beispiele muss jedoch mit Bedauern konstatiert werden, dass es sich hierbei vor allem um negative,

8 Der TV-Film „Unsere Mütter, unsere Väter“ erzählt die Geschichte einer Gruppe junger Deutscher im Zweiten Weltkrieg. Mit dem Film wollten die Produzenten Tabus brechen und intergenerationelle Gespräche über die Vergangenheit in Gang bringen. Die gezielte Vermischung von Dokument und Fiktion sowohl im Film als auch in begleitenden Dokumentationen sollte Authentizität und Wirklichkeitstreue suggerieren.

9 Der Film wurde in über 80 Länder verkauft und u. a. mit einem International Emmy Award 2014 als beste Miniserie ausgezeichnet. 
abwertende und stereotypisierende Bilder und Sichtweisen handelt. Bestimmte, an sich schädliche und oft ungerechte Vorstellungen, Muster, Schemata, Stereotype usw. werden instrumental benutzt und je nach Bedarf (bzw. politischen Interessen) eingesetzt. Dadurch werden sie nicht abgeschafft, sondern konserviert und stabilisiert.

Es ist wohl eine allgemeine (massenmediale) Strategie der postfaktischen Politik, die Grenzen des Sagbaren und des Zeigbaren hinauszuschieben, d. h., sich verbal und ikonisch gegen den gesellschaftlichen Konsens zu verhalten: „Fakten sind unwesentlich geworden; viel wichtiger sind Gefühle und Wünsche. Entscheidend ist nicht das, was sich tatsächlich ereignet hat, sondern das, was man befürchtet." (Wolf, 2017, S. 2) Im analysierten Fall geht es nicht nur um die Grenze zwischen Wahrheit und Lüge, sondern auch um Wörter und Bilder, die man angesichts der deutsch-polnischen Geschichte nicht mehr bzw. nie in dieser Weise verwenden sollte.

Viele Redaktionen müssen sich in diesem Zusammenhang den Vorwurf gefallen lassen, dass sie ganz bewusst mit einem ,Reizwort oder -bild' bzw. einem multimodalen ,Reiz-Kommunikat' keine Sachverhalte benennen bzw. zeigen, sondern in erster Linie Emotionen ihrer Rezipient*innen erregen wollen.

\section{Fazit}

Emotionen sind interpretative Konstruktionen auf der Grundlage sichtbarer Elemente - Konglomerate von Zeichen unterschiedlicher Modalität, die miteinander zusammenwirken. Intermodale Kommunikate wie z. B. Titelseiten von Nachrichtenmagazinen können demnach als Generatoren von Emotionen betrachtet werden.

In Kombination mit entsprechend sprachlich vermittelten Angaben und/oder linear-rationaler Argumentation können z.B. Bilder ihre emotionskonstruierende Wirkmächtigkeit entfalten und diskursbezogen kommunikative Perspektivierungen anzeigen (vgl. Meier, 2011, S. 514). Aus der sichtbaren Anordnung von wiedererkennbaren Elementen auf der Bildbzw. Sehfläche resultieren ein bestimmtes Sinnpotenzial und ein mindestens gleich relevantes Emotionspotenzial, das „mit einer spezifischen Überzeugungskraft, einer Form anschaulicher Evidenz" (Steinseifer, 2011, S. 4) verbunden ist.

Aus Sicht einer diskursanalytisch fundierten, medialitätsorientierten Emotionsforschung kann Emotionalisierung der Medieninhalte als eine 
komplexe kommunikative Handlung angesehen werden, die von Diskursakteuren unter Einsatz diverser Modi vollzogen wird. Emotionalisieren hingegen ist ein Diskursmuster, in dem verschiedene Modalitäten verwendet werden.

Emotionen als multimodale, diskursiv konstruierte Phänomene können die kollektive Bewusstseinsbildung und Handlungsorientierung beeinflussen, das kollektive Gemeinschaftsgefühl und kollektive Gedächtnis stärken und nicht selten affektive nationalgeprägte bzw. sichtweisenspezifische Weltbilder kreieren. Sie werden - oft manipulativ - zur Herstellung „affektiver Öffentlichkeiten“ (Papacharissi, 2015) bzw. „Wut- und Empörungsgemeinschaften“" (Pörksen \& Detel, 2012) eingesetzt.

\section{LITERATURVERZEICHNIS}

Bendel Larcher, S. (2015). Linguistische Diskursanalyse. Eine Einführung. Tübingen: Narr.

Bucher, H.-J. (2012). Multimodalität - ein universelles Merkmal der Medienkommunikation: Zum Verhältnis von Medienangebot und Medienrezeption. In: H-J. Bucher \& P. Schumacher (Hrsg.), Interaktionale Rezeptionsforschung. Theorie und Methode der Blickaufzeichnung in der Medienforschung (S. 51-82). Wiesbaden: Springer.

Busse, D. (2016). Einführung: Kulturwissenschaftliche Orientierung in der Sprachwissenschaft, In: L. Jäger, W. Holly, P. Krapp, S. Weber \& S. Heekeren (Hrsg.), Sprache - Kultur Kommunikation. Ein internationales Handbuch zu Linguistik als Kulturwissenschaft (S. 645-661). Berlin, Boston: de Gruyter.

Eckkrammer, E. \& Held, G. (Hrsg.) (2006). Textsemiotik. Studien zu multimodalen Texten. Frankfurt a. M.: Lang.

Felder, E. (2012). Pragmasemiotische Textarbeit und der hermeneutische Nutzen von Korpusanalysen für die linguistische Mediendiskursanalyse. In: E. Felder, M. Müller \& F. Vogel (Hrsg.), Korpuspragmatik. Thematische Korpora als Basis diskurslinguistischer Analysen (S. 115-174). Berlin, Boston: de Gruyter.

Foucault, M. (2001a). Worte und Bilder. In: D. Defert, F. Ewald \& J. Lagrange (Hrsg.), Michel Foucault. Schriften in vier Bänden. Dits et Ecrits. Bd. 1 1954-1969 (S. 794-797). Frankfurt a. M.: Suhrkamp.

Foucault, M. (2001b). Antwort auf eine Frage. In: D. Defert, F. Ewald \& J. Lagrange (Hrsg.), Michel Foucault. Schriften in vier Bänden. Dits et Ecrits. Bd. 1 1954-1969(S. 859-886). Frankfurt a. M.: Suhrkamp.

Fricke, E. (2012). Grammatik multimodal: Wie Wörter und Gesten zusammenwirken (Linguistik - Impulse \& Tendenzen, 40, Band 40) (1. Aufl.). Berlin, New York: De Gruyter. https://doi.org/10.1515/9783110218893

Gebauer, G. (2009). Wittgensteins anthropologisches Denken. München: C.H. Beck.

Hess-Lüttich, E. W. B., Kämper, H., Reisigl, M. \& Warnke, I. H. (2017). Vorwort. In: E.W.B. Hess-Lüttich, H. Kämper, M. Reisigl \& I. H. Warnke (Hrsg.), Diskurs semiotisch. Aspekte multiformaler Diskurskodierung (S. VII-X). Berlin, Boston: de Gruyter. https://doi.org/10.1515/9783110489057 
Igl, N. \& Menzel, J. (2016). Einleitung. Zur medialen Eigenlogik Illustrierter Zeitschriften. In: N. Igl,\& J. Menzel (Hrsg.): Illustrierte Zeitschriften um 1900: mediale Eigenlogik, Multimodalität und Metaisierung (S. 11-20). Bielefeld: transcript.

Keller, R. (2003). Zu einer Theorie der semiotischen Kompetenz. In: A. Linke, H. Ortner \& P. R. Portmann-Tselikas (Hrsg.), Sprache und mehr. Ansichten einer Linguistik der sprachlichen Praxis (S. 165-175). Tübingen: Niemeyer.

Keller, R. (2005). Wissenssoziologische Diskursanalyse. Grundlegung eines Forschungsprogramms. Wiesbaden: Springer VS. https://doi.org/10.1007/978-3-53192058-0

Keller, R. (2013). Zur Praxis der Wissenssoziologischen Diskursanalyse. In: R. Keller \& I. Truschkat (Hrsg.), Methodologie und Praxis der Wissenssoziologischen Diskursanalyse. Band 1: Interdisziplinäre Perspektiven (S. 27-68). Wiesbaden: VS Verlag für Sozialwissenschaften. https://doi.org/10.1007/978-3-531-93340-5

Klug, N.-M. (2018). Wenn Schlüsseltexte Bilder sind. Aspekte von Intertextualität in Presse und öffentlichem Raum. In: S. Pappert \& S. Michel (Hrsg.), Multimodale Kommunikation in öffentlichen Räumen. Texte und Textsorten zwischen Tradition und Innovation (S. 109-132). Stuttgart: ibidem.

Knoblauch, H. \& Tuma, R. (2018). Wissen. In: J. Kopp \& A. Steinbach (Hrsg.), Grundbegriffe der Soziologie (12. Aufl., S. 517-519). Wiesbaden: Springer VS. https://doi.org/10.1007/978-3-658-20978-0_100

Massen, S., Mayerhauser, T. \& Renggli, C. (2006). Bild-Diskurs-Analyse. In: S. Massen, T. Mayerhauser \& C. Renggli (Hrsg.), Bilder als Diskurse - Bilddiskurse (S. 7-26). Göttingen: Velbrück Wissenschaft.

Meier, S. (2011). Multimodalität im Diskurs: Konzept und Methode einer multimodalen Diskursanalyse. In: R. Keller, A. Hirseland, W. Schneider \& W. Viehöver (Hrsg.), Handbuch Sozialwissenschaftliche Diskursanalyse. Bd. 1: Theorien und Methoden (3., erweit. Aufl., S. 499-532). Wiesbaden: VS.

Papachrissi, Z. (2015). Affective publics: sentiment, technology and politics. Oxford: Oxford University Press. https://doi.org/10.1093/acprof:oso/9780199999736.001.0001

Pörksen, B. \& Detel, H. (2012). Der entfesselte Skandal. Köln: Herbert von Halem Verlag.

Renggli, C. (2014). Komplexe Beziehungen beschreiben. Diskursanalytisches Arbeiten mit Bildern. In: F. X. Eder, O. Kühschelm \& Ch. Linsboth (Hrsg.), Bilder in historischen Diskursen. Interdisziplinare Diskursforschung (S. 45-62). Wiesbaden: Springer Fachmedien. https://doi.org/10.1007/978-3-658-05398-7

Sachs-Hombach, K. \& Schirra, J. R. J. (2009). Medientheorie, visuelle Kultur und Bildanthropologie. In: K. Sachs-Hombach (Hrsg.), Bildtheorien. Anthropologische und kulturelle Grundlagen des Visualistic Turn (S. 393-427). Frankfurt a. M.: Suhrkamp.

Schiewer, G. L. (2014). Studienbuch Emotionsforschung. Theorien - Anwendungsfelder Perspektiven. Darmstadt: WBG.

Spitzmüller, J. \& Warnke, I. H. (2011). Diskurslinguistik. Eine Einführung in Theorien und Methoden der transtextuellen Sprachanalyse. Berlin, Boston: de Gruyter.

Steinseifer, M. (2011). Terrorismus" zwischen Ereignis und Diskurs. Zur Pragmatik von TextBild-Zusammenstellungen in Printmedien der 70er-Jahre. Berlin, Boston: de Gruyter. https://doi.org/10.1515/9783110229387

Traue, B. (2014). Resonanz-Bild und ikonische Politik. Eine visuelle Diskursanalyse partizipativer Propaganda. In: M. Kauppert \& I. Leser (Hrsg.), Hillarys Hand. Zur politischen Ikonographie der Gegenwart. (S. 131-156). Bielefeld: transcript. 
Wolf, N. R. (2017). Sprechen und Sprache in der postfaktischen Politik. Sprachreport, 1/2017, 1-6. 